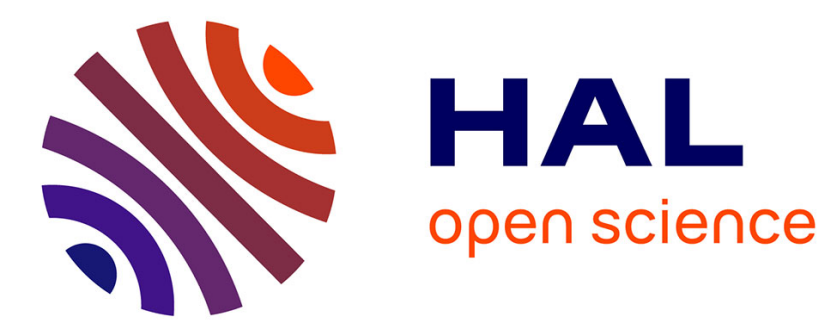

\title{
Power-Aware Population Protocols
}

\author{
Chuan Xu, Janna Burman, Joffroy Beauquier
}

\section{To cite this version:}

Chuan Xu, Janna Burman, Joffroy Beauquier. Power-Aware Population Protocols. 2017 IEEE 37th International Conference on Distributed Computing Systems (ICDCS), Jun 2017, ATLANTA, United States. 10.1109/ICDCS.2017.238 . hal-01458930v3

\section{HAL Id: hal-01458930 \\ https://hal.science/hal-01458930v3}

Submitted on 9 Apr 2017

HAL is a multi-disciplinary open access archive for the deposit and dissemination of scientific research documents, whether they are published or not. The documents may come from teaching and research institutions in France or abroad, or from public or private research centers.
L'archive ouverte pluridisciplinaire HAL, est destinée au dépôt et à la diffusion de documents scientifiques de niveau recherche, publiés ou non, émanant des établissements d'enseignement et de recherche français ou étrangers, des laboratoires publics ou privés. 


\section{Power-Aware Population Protocols}

\author{
Chuan $\mathrm{Xu}$ \\ Université Paris Saclay \\ LRI, France \\ Email: chuan.xu@1ri.fr
}

\author{
Janna Burman \\ Université Paris Saclay \\ LRI, France \\ Email: janna.burman@1ri.fr
}

\author{
Joffroy Beauquier \\ Université Paris Saclay \\ LRI, France \\ Email: joffroy.beauquier@lri.fr
}

\begin{abstract}
The contribution of the paper is twofold. First, it introduces a model for analyzing energy consumption in networks of mobile sensors. Second, it uses this model for studying energy complexity of distributed protocols for the task of data collection. The proposed model is quite general and can be used for other tasks. It can be considered as the first extension of this kind, taking into account energy consumption, of the classical model of population protocols. In population protocols, anonymous and bounded memory agents (sensors) move unpredictably and communicate in pairs when two of them are in proximity. The interest of the extended model is to allow a purely analytical analysis of the energy complexity of a protocol, in the same spirit as for time and space complexity, without appealing to simulations. This approach allows to exhibit energy functions and to draw their curves, from which the optimal values of various parameters can be deduced.
\end{abstract}

In order to illustrate the power and the usefulness of this model, we consider the issue of determining and adjusting the amount of agents' initial energy necessary and sufficient for being able to perform a given task. This issue is crucial for choosing, in practice, a category of sensors (in respect with their power capacities) adapted both to the task and to the number of times it should be repeated (before the sensors are replaced or recharged). In this context, the natural chosen metric is the maximum energy spent by an agent (for accomplishing the overall collaborative task). This metric is directly related to the minimum necessary initial energy (and also to the lifetime of the network). Contrary to most of the energy studies in networks of mobile agents, our approach is completely deterministic. The reason is that due to the nature of the considered problem, the analysis has to be done in the worst case (in particular, such analysis is impossible when the interactions between agents are supposed to be probabilistic).

The specific task we consider in this work is data collection, which is known to be fundamental in sensor networks. In this problem, initially, each sensor has an input (a sensed) value, and eventually, every input should be delivered exactly once, to a base station. Transfers of inputs between sensors are possible in order to optimize time and energy metrics. In this context, our first contribution is the energy complexity analysis of an already known time optimal protocol. The second contribution is a new power-aware protocol, which improves the previous one in terms of the maximum energy spent by an agent. Finally, we present a lower bound concerning energy consumption of any possible data collection protocol and we show the cases where this lower bound is reached by the presented protocols.

Index Terms-mobile sensor networks; population protocols; energy consumption; data collection

\section{INTRODUCTION}

In wireless mobile networks, routing is a complex problem, mainly due to node mobility resulting in a frequently changing network topology. The rate of the topology changes depends on many factors including node mobility patterns and speeds, and characteristics of the terrain and of the communication medium. Typical metrics used to evaluate routing protocols like shortest-hop, shortest-delay, and local stability may have a negative effect on the network performance, because they result in an overuse of energy resources of a small set of nodes, decreasing network lifetime. Consequently, it is essential to consider algorithms from a power-aware perspective, in addition to such traditional metrics.

In this work, we are interested in a special type of mobile sensor networks, where the sensors are extremely simple and cheap. They have very limited computing and communication capabilities. They have no identifiers and execute all the same code. The size of their memory is uniformly bounded, independently of the total number of sensors in the network. As their communication power is limited, they do not communicate by broadcasting, but only, in pairs, when two agents come close to each other. Moreover, the number of nodes in the network is unknown and may be very large. Though, they generally move in a limited area. ${ }^{1}$

In this framework of very simple mobile sensors, we consider a restricted, but natural (for sensor networks), form of routing toward a special node, called data collection. One may assume that each sensor has got a value from the environment (temperature, pressure, altitude, etc.), and that all values must be routed towards a base station, where they will be analyzed and treated. As the considered mobile sensors interact unpredictably and only in pairs, the data collection is by essence opportunistic, meaning that it has to be performed even though there are no permanent routes from mobile sensors to the base station. Then, when a node meets another node, it has to decide, according to its protocol, whether it is better or not to transfer its sensed value to the other or to keep it. Better usually refers to the global time for performing data collection.

The issue that we address here is how to route the sensors' values towards the base station and spend as little energy as possible, while retaining good performances in terms of data collection time. Our approach is at the algorithmic level. It means that, beside the classical time complexity, we define formally an energy complexity, the goal being to compute time and energy complexities in a purely analytical way, independent of simulations.

\footnotetext{
${ }^{1}$ As examples to such networks, one can consider the ZebraNet project deploying a network of sensors attached to zebras [1] or the Pigeon Air Patrol Network project, with pigeons carrying very simple sensors.
} 
The distributed computing model that we choose for the considered networks is the (classical) model of population protocols (PP) [2]. This model has been designed to study asynchronous networks of finite state anonymous mobile sensors (called agents) interacting in pairs. We enhance this model with an energy consumption framework. Basically, an agent consumes energy when: (1) interacting and exchanging data, and (2) signaling its presence and sensing the wireless medium continuously (to allow detection of other agents in proximity). An interacting agent is said to be in awake mode and spends an amount $E_{w k p}$ of energy. When it does not interact, but senses the medium, it is in sleep mode, and spends $E_{s l p}$ for every global interaction (that can be seen as a time unit). We also consider an additional mode called terminated, in which an agent consumes no energy. This mode can allow saving energy of agents having terminated their part in a task.

To adapt this general model to the specific type of networks we consider here, we make some additional considerations and assumptions detailed in Sect. II. For instance, as the data exchanged during an interaction is bounded by the small agent's memory, we can assume that any transmitted data fits in one packet and thus the energy spent in a communication is constant. In addition, as the agents communicate only in pairs when they come close to each other, it is likely to assume that the sensing of the medium is done by proximity sensors (cf. [3], [4]) and thus, agents signal their presence only passively, spending no energy.

In this study, we analyze the maximum amount of energy spent by an agent for performing a one-time collaborative task (data collection in our case). This metric is clearly related to the lifetime of the network and to the amount of the required initial energy for each agent. The formal analysis of this metric allows, in advance, to adjust the network both in terms of size and battery power allocated to the agents. It is particularly important in the case of networks where it is difficult or undesirable to access the sensors frequently (cf., Bird Species Recognition [5]). However, it is important to note that, if no guarantees are imposed on the agents' interactions, such analysis is in general impossible (for non-trivial protocols). Consider, for example, one of the classical assumptions in PP where any pair of agents is required to interact only infinitely often. In this case, protocols converge only eventually, consuming an arbitrary large energy till convergence. On the contrary, with another classical assumption where pairs of agents interact according to some probability distribution, an average complexity analysis of energy consumption can be computed.

Here, for having a bounded convergence and still being able to perform a worst case analysis of the maximum energy spent, we do not adopt the probabilistic approach, but we assume a sort of partial synchrony. According to it, an agent interacts with all the others with a certain "frequency", expressed by a cover time parameter [6]. This is an upper bound on the "time", counted in number of global interactions (pairwise meetings), for an agent to interact with all the others. The "faster" an agent meets the others, the smaller its cover time is. ${ }^{2}$

As shown by recent experimental and analytical studies [10], [11], [12], [13], the assumption of bounded cover times is (maybe surprisingly) well adapted for modeling mobility in practical sensor networks. The relevant networks are those where agents have different mobility and communication capabilities, and move within a bounded area or have tendency to return periodically to some specific places, e.g., their homes. The experiments studied networks of students in a campus, participants in a network conference, visitors at Disney World and more [10], [14], [15]. The analytical studies of such networks show that the inter-contact time of two specific agents is practically bounded. Since a cover time of an agent can be expressed as a sum of inter-contact times of the agent with the others, bounded cover times can model well the mobility in such networks.

Contributions. The main general contribution of this paper is the framework allowing an analytical computation of energy consumption, presented for the first time in the context of population protocols (Section II). Using this energy model, we start by analyzing energy consumption of the data collection protocol proposed and proven to be time optimal in [6] (Section III). In this analysis, our approach is motivated by the observation that the energy spent is proportional to the length of an execution. However, further investigation shows that this is not the only factor that can affect the energy spent, and other factors, as the residual energy of an agent, if considered by a solution, can improve energy performance. This gives an idea for developing a better solution in terms of energy consumption, and we propose an energy-aware protocol. We prove its correctness and analyze its energy complexity (Section IV-A and IV-B). Not surprisingly, it appears that it needs less energy than the first protocol when correctly adjusting the protocol's parameters. Having analytic formulas for energy consumption under the form of functions allows to draw graphs and plots, on which remarkable points, zones and limits appear immediately (Sect. IV-B2 and IV-B3). This is particularly advantageous when a great number of parameters are involved and when the exhibited functions are hybrid (i.e., composed of several functions). Getting the same or approximative results from simulations would certainly be longer and costly, while here exact results can be obtained almost immediately. The paper presents such plots as an illustration of this methodology. Section V presents a lower bound concerning energy consumption of any possible data collection protocol. Cases where this lower bound is reached by the presented protocols are also given. Finally, Sect. VI is

\footnotetext{
${ }^{2}$ The notion of cover time has some similarities with partial synchrony [7] or timeliness [8] (in a lock-step message-passing or shared-memory communication model), in the sense that the cover times impose constraints on the relative (activation) speeds and on the communication of processes (here agents). This sort of synchrony is partial, because the agents have no access to the cover time values.

In the context of population protocols, somewhat similar to cover times' guarantees on agents' interactions are assumed in the implementation of the cover time service proposed in [9], for augmenting population protocols with termination detection.
} 
a summary of the paper results.

Note that, in most of the study, we consider the more difficult, but also more realistic case, where an agent can hold only a limited number of values (independent of the number of agents). Such an assumption is coherent with the spirit of population protocols.

Related Work. There is a very large literature on power-aware network protocols. The reader could find general references in surveys, like [16], [17] for power-aware routing protocols in MANET, like [18] for data collection in mobile sensor networks, and like [19] for optimistic routing in highly dynamic networks. Most of the works on energy consumption do not present an analytical study of energy complexity, and the validation of the approaches is made by simulation. In this section, we will only mention the studies directly linked to our approach by restricting our attention to those for which a formal model of energy consumption is presented and its mathematical analysis is done.

In this category, we should first mention [20] where authors study the problem of energy balancing between non finite state agents (interacting uniformly at random) in a model inspired by population protocols. The considered model is not an energy consumption one. An interacting agent can transfer some part of its energy to the other agent during the interaction (by using a corresponding energy equipment) and there may be a constant energy loss for each unit of the transferred energy. However, contrary to our study, energy consumption for interacting and sensing is not considered. Three protocols, for balancing (averaging) the distribution of energies given to agents, are presented and their analytical or simulation analysis is presented.

Then, one can mention [21], which studies a synchronized scheduling of wake-up times for improving the data collection in a non-mobile sensor network. It proposes protocols with energy consumption of at most twice the optimum. Synchronization of the wake-up times is also studied in [22], where several self-stabilizing protocols to solve the problem of temporal partition are presented and analyzed.

In [23], a 2-approximation (energy consumption) centralized heuristic and a distributed power-aware heuristic are developed for wireless sensor networks with unique identifiers, to ensure that all packets are delivered with the minimum energy cost within a required deadline. Authors develop an evaluation function of energy cost for one transmission, considering residual energy, quality of link and the type of nodes.

In [24], authors propose a randomized clustering algorithm for non-mobile wireless sensor networks which minimizes the energy spent for transmissions. It is proven formally that the algorithm has a time complexity of much less than $O(n)$.

In [25], authors give an approximated cone-based topology scheme to increase ad-hoc network lifetime with adaptive transmission power control, while maintaining connectivity for routing. In an analytical way, it is shown that the power consumption of each route can be made arbitrarily close to the optimal.
[26] studies the performance of a class of simple and local algorithms for energy-efficient construction of minimum spanning trees in a wireless ad hoc setting. Bounds on the performance of these algorithms, in instances obtained by uniformly distributed points in the unit square, are given.

For being complete, we should also mention that there exist studies presenting a mathematical model for optimizing the energy-consumption of a single entity, independently of the protocol run, but they are very far from our preoccupations.

Finally, even if some approaches considering energy consumption are in spirit similar to ours, none of them is devoted to the specific type of networks that we consider: anonymous resource-limited mobile sensors moving unpredictably and constituting a highly dynamic opportunistic network.

\section{Model And Notations}

\section{A. Population Protocols (PP)}

A system consists of a collection $\mathcal{A}$ of pairwise interacting agents, also called a population. Each agent represents a finite state sensing and communicating mobile device. Among the agents, there is a distinguishable agent called the base station (BST), which can be as powerful as needed, in contrast with the resource-limited non-BST agents. The non-BST agents are also called mobile, interchangeably. The size of the population $|\mathcal{A}|=n$ is unknown to the agents.

A (population) protocol can be modeled as a finite transition system whose states are called configurations. A configuration is a function that associates each agent with its state. Each agent has a state taken from a finite set of states, the same for all non-BST agents, but generally different for BST. Every transition $C \rightarrow C^{\prime}$ between two configurations $C$ and $C^{\prime}$ is modeled by a single transition between two agents happening during an interaction. That is, when two agents $x$, in state $p$, and $y$, in state $q$, in $C$, interact (meet), they execute a transition rule $(p, q) \rightarrow\left(p^{\prime}, q^{\prime}\right)$. As a result, in $C^{\prime}, x$ changes its state from $p$ to $p^{\prime}$ and $y$ from $q$ to $q^{\prime}$.

An execution of a protocol is a sequence of configurations $C_{0}, C_{1}, C_{2}, \ldots$ such that $C_{0}$ is the starting configuration and for each $i \geq 0, C_{i} \rightarrow C_{i+1}$. In a real distributed execution, interactions could take place simultaneously, but when writing down an execution we can order those simultaneous interactions arbitrarily. An execution is said to be finite, iff from some point on, no applicable transition changes the configuration. In this case, this non-changing configuration is said to be terminal. When a terminal configuration is reached, we say that the termination has occurred. Each execution corresponds to a unique sequence of interactions, also called schedule. If an execution $e$ is finite, its length $|e|$ is the number of interactions until termination. For convenience, we consider the number of interactions in an execution as the time reference, i.e., each next interaction adds one time unit to the global time.

Cover Time Fairness. Every agent $i \in \mathcal{A}$ has (an unknown to agents) parameter $c v_{i} \in \mathcal{N}$ called cover time s.t. during any $c v_{i}$ time units (consecutive interactions in an execution), agent $i$ interacts with every other agent at least once. $c v_{i}$ is the minimum such number of interactions. For two agents $x$ 
and $y$, if $c v_{x}<c v_{y}$, then we say that $x$ is faster than $y$, and $y$ is slower than $x$. The minimum cover time value is denoted by $c v_{\min }$ and the maximum one by $c v_{\max }$. A fastest (resp. slowest) agent $z$ has $c v_{z}=c v_{\min }$ (resp. $c v_{z}=c v_{\max }$ ). We denote by $F$ the set of fastest mobile agents, and by $N F$ the set of non-fastest ones.

In the protocol analysis, we consider only the case where $c v_{B S T}>c v_{\text {min }}$, that maximizes time and energy consumption (for data collection). In some cases, for simplicity, we also assume that $c v_{\min } \gg O\left(n^{2}\right)$. This assumption holds for a large and natural family of cover time vectors. It is especially natural for the case of passively mobile sensor networks, which is exactly the type of networks modeled by population protocols. Intuitively, as $c v_{\text {min }}$ (in this family) is much larger than the number of all possible pair-wise interactions, agents are free to interact in many different ways, as it would be naturally expected with passive mobility. Still very particular schedules are possible and there are guarantees that agents interact according to the cover times.

We emphasize that agents are not assumed to know cover times (to conform with the finite state population protocol model). Instead, we do assume that two interacting agents can only compare their respective cvs. As described in [6], this can be implemented with finite state agents, if the number of different $c v$ s is finite too.

Data Collection. A problem is defined by a predicate $\mathcal{D}$ on executions. A population protocol $\mathcal{P}$ is said to solve a problem $\mathcal{D}$, if and only if every execution of $\mathcal{P}$ satisfies the conditions defining $\mathcal{D}$.

The problem of data collection (or data gathering) is defined as follows. We assume that each agent, except BST, owns initially an input value (which is constant during one execution of the protocol). Eventually, every input value has to be delivered to BST, and exactly once (as a multi-set). When this happens, we say that the protocol (its execution) has converged or terminated. The convergence time of a protocol is the maximum length of a possible execution (until convergence). We denote by $M$ the number of values that a non-BST agent can receive from other agents (on top of its own initial value). When describing or analyzing a considered protocol, the term "to transfer a value" from agent $x$ to $y$ means to copy it to $y$ 's memory, and erase it from the memory of $x$.

\section{B. Energy Consumption Scheme and Metrics}

During an execution, each non-BST agent consumes energy according to three different modes: sleep, awake and terminated. The overall consumption scheme can be summarized by the following points:

1) Each agent starts with the same amount of initial energy $E_{0}$ (e.g., all agents are fully charged). It is in sleep mode, and consumes $E_{s l p}$ per time unit (every interaction). During this mode, an agent consumes a reduced amount of energy. It senses the vicinity for detecting other agents in proximity, and signals its presence. The signalling can be done either actively, by emission of small beacon messages (as, e.g., in IEEE 802.11), or passively, only by agent's presence. The latter can be carried out by sensing the vicinity using proximity sensors (cf. [3], [4]). In this work, we consider this latter case of sensing.

2) When two agents in sleep mode have successfully detected each other, the interaction happens and both turn into awake mode to proceed with a communication. For that, they have to switch on their radio transceivers, which is generally a high energy consuming operation (cf. [27]). During the interaction, both agents stay awake and each consumes $E_{w k p}=E_{s w}+E_{t x}+E_{r x}+$ $E_{s l p}$, where $E_{s w}$ is the energy consumed for switching to the main radio, $E_{t x}$ and $E_{r x}$ are consumed during the sending and the reception of data, and $E_{s l p}$ is due to point 1 .

Since in the considered types of networks (and in PP) non-BST agents have a small memory, independent of the network size, transmitted data can fit in very few, and even one, packets in every communication. Thus, we can assume that the energy consumed by the communication, $E_{t x}+E_{r x}$, is constant.

3) During an interaction, two awake agents decide to turn either to sleep or to terminated mode, according to the protocol. No energy is consumed by a terminated agent. This mode is useful for saving energy of agents that have terminated their protocol activities. Together with that, the scheme can be adapted in such a way that a terminated agent could be restored into sleep mode, whenever it is required to restart a task. This can be done, for example, by a global signal from BST, which, at the same time, can also recharge the mobile agents, to prepare them for the next task [28]. ${ }^{3}$

4) We assume that when a sleeping agent $x$ meets a terminated agent $y, x$ wakes up and thus, spends $E_{w k p}$. This assumption makes sense in networks deploying proximity sensors for sensing close agents. In this case, the terminated agent $y$ is detected by the proximity sensor, and as $x$ is not aware of $y$ 's mode in this step, $x$ wakes up and only then detects that $y$ is terminated. ${ }^{4}$

Evaluation Metrics. There are many different power-aware metrics in the related literature on sensor networks. The term lifetime of a network is generally used and can be evaluated, e.g., as the maximum time until only one or all sensors have no energy, or until there are more than a ratio $\beta$ of surviving nodes, or until "connectivity" is lost. Alternatively, to analyze the lifetime, one can evaluate the maximum energy spent per node for a given task (e.g., per data collection from all nodes to BST, or per packet routing to the next node, or to the final destination). Refer to the surveys on such metrics [29], [30].

In this work, we choose to evaluate and minimize the maximum energy spent per node across the network in each data collection, thereby enhancing the lifetime performance. Given

\footnotetext{
${ }^{3}$ In this work, we do not consider this possibility, as we analyze energy consumption for only one data collection.

${ }^{4}$ However, with a beacon based approach for agents' detection (described in the first point), neither $x$, nor $y$ spend $E_{w k p}$ in such an interaction.
} 
a protocol $\mathcal{P}$, we denote by $E s_{\max }(\mathcal{P})$ the maximum energy spent by an agent in all executions of $\mathcal{P}$. This metric is one of the most popular metrics studied in power-aware routing protocols for MANET [30]. Minimizing it can be also seen as balancing the distribution of energy consumption among agents. Moreover, as already mentioned in the introduction, evaluation of this metric can allow, in advance, to adjust the network both in terms of size and battery power allocated to the agents.

\section{ENERGy CONSUMPTION OF TTFM}

In this section, we consider an existing protocol, which was designed without energy consumption in mind, and we perform its energy consumption analysis, using the proposed energy model. TTFM (Transfer To the Faster Marked) is a time-optimal protocol for data collection in PP [6]. The basic strategy for data transfer, is that a mobile agent only transfers its values either to BST, or to a faster agent that has never met (yet) another mobile agent faster than itself. When two fastest agents meet, there is no transfer of data. The idea of TTFM is to make use of the fastest agents, which are more likely to bring the values faster to BST.

For illustration of the usage of terminated mode, we adapt TTFM such that a mobile agent turns into terminated mode once it has transferred its values to a faster agent (excluding BST). Indeed, according to TTFM, once this happens, the slower agent does not receive any value till the end of the execution. Therefore, turning into terminated mode saves energy. For the fastest agents, as they never transfer values to other agents except to BST, they stay non-terminated until convergence. This adapted protocol, called E-TTFM, is presented below (Protocol 1).

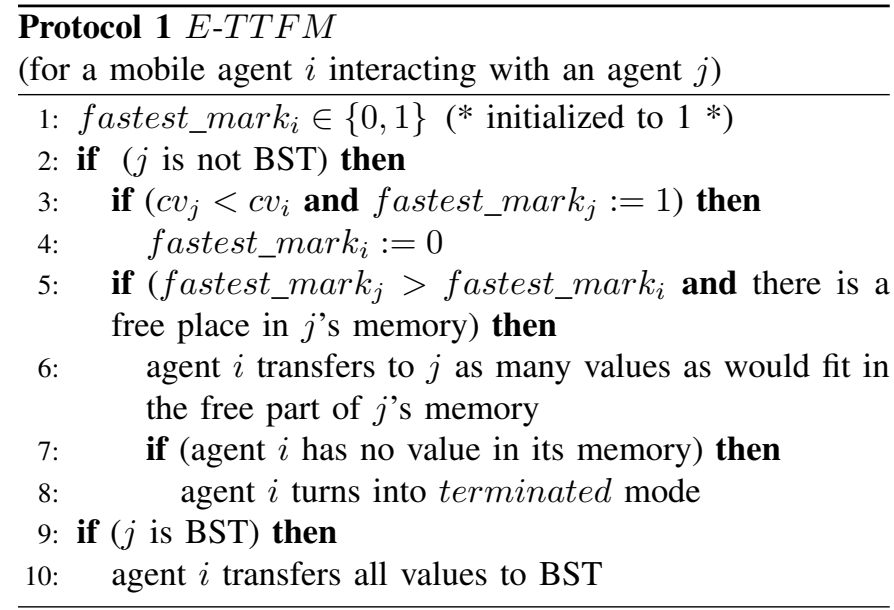

$E-T T F M$ has the same time complexity as TTFM, because the adaption we did does not influence the worst case convergence time of data collection. Therefore, the longest execution in $E$-TTFM is of length $2 c v_{\min }\left\lceil\frac{|N F|}{M|F|}\right\rceil-1$ (Th. 11 in [6]). The worst case on the maximum energy spent by an agent is attained in the longest execution, in which one fastest agent participates in a maximum number of interactions (being in awake mode as long as possible). This implies the following result:

Proposition 1: An upper bound on the maximum energy spent by an agent in $E-T T F M$ is $\left(2 c v_{\min }\left\lceil\frac{|N F|}{M \times|F|}\right\rceil-1\right) E_{w k p}$. This bound is reached when $c v_{\min } \gg O\left(n^{2}\right)$.

Proof: The upper bound is easy to obtain, as the longest execution in $E-T T F M$ is of length $\left(2 c v_{\min }\left\lceil\frac{|N F|}{M \times|F|}\right\rceil-1\right)$. Thus, no agent can consume more than $\left(2 c v_{\min }\left\lceil\frac{|N F|}{M \times|F|}\right\rceil-\right.$ 1) $\times E_{w k p}$ energy.

Next, we prove that this bound can be reached when $c v_{\min } \gg$ $O\left(n^{2}\right)$. Consider the following schedule which repeats the segment $X_{1} X_{2}$, where $X_{1} X_{2}$ is of length $c v_{\text {min }}$. We denote by $f$ to be one of the fastest agents.

$X_{1} \equiv[$ repeat as much as possible all interactions between $f$ and every agent in $A \backslash \mathrm{BST}$ ],

$X_{2} \equiv[$ all the other necessary interactions to satisfy cvs ].

Consider the execution of E-TTFM for this schedule, denoted by $e$. This execution could be the worst case execution in time presented in [6]. Let us choose such $e$ and thus, $|e|=\left(2 c v_{\min }\left\lceil\frac{|N F|}{M \times|F|}\right\rceil-1\right)$.

In $E-T T F M$, a fastest agent never turns to terminated. Therefore, in $e$, agent $f$ consumes the most $\left(E_{w k p}\right.$ energy in each interaction of $\left.X_{1}\right)$. Thus, $E s_{\max }(e)=(|e|-$ $\left.\epsilon\left|X_{2}\right|\right) E_{w k p}+\epsilon\left|X_{2}\right| E_{\text {slp }}$ where $\epsilon\left|X_{2}\right|$ is a constant indicating the number of necessary interactions in $X_{2}$ not involving the agent $f$ in $e$.

Notice that $\left|X_{2}\right| \leq \frac{n(n-1)}{2}=O\left(n^{2}\right)$ and as $c v_{\min } \gg O\left(n^{2}\right)$, the length of $X_{2}$ is negligible. Therefore, when $c v_{\min } \gg$ $O\left(n^{2}\right), E s_{\max }(E-T T F M)=E s_{\max }(e)=|e| E_{w k p}=$ $\left(2 c v_{\min }\left\lceil\frac{|N F|}{M \times|F|}\right\rceil-1\right) E_{w k p}$.

\section{ENERGY-BALANCEd Protocol EB-TTFM}

A first step towards a protocol balancing the distribution of energy consumption would be to consider the following strategy: When an agent notices that it becomes "low" in energy, it tries to transfer its values to a higher energy level agent and turns into terminated mode, as soon as possible. The issue raised by this strategy is that the convergence time for data collection could be possibly considerably augmented, especially if some values are transferred to a slow agent. An increased convergence time means more energy spent.

Therefore, the level of the amount of residual energy that is considered to be "low" should be carefully set. Below, we discuss the possible choices of such a level. We make these choices depend on a parameter, $\lambda$, by defining the "low" level by $\frac{E_{0}}{\lambda}$ where $\lambda \geq 1$. To conform with the finite state population protocol model, we assume that $\frac{E_{0}}{\lambda}$ can only take a finite number of values. Let $e l_{i}$ be the residual energy of the mobile agent $i$. In the same way as for cover times, we assume that the agents do not know their residual energies but can compare them when they interact. Adding the test of the energy level to $E-T T F M$ yields a protocol that we name EB-TTFM (for Energy Balanced TTFM, Protocol 2). 


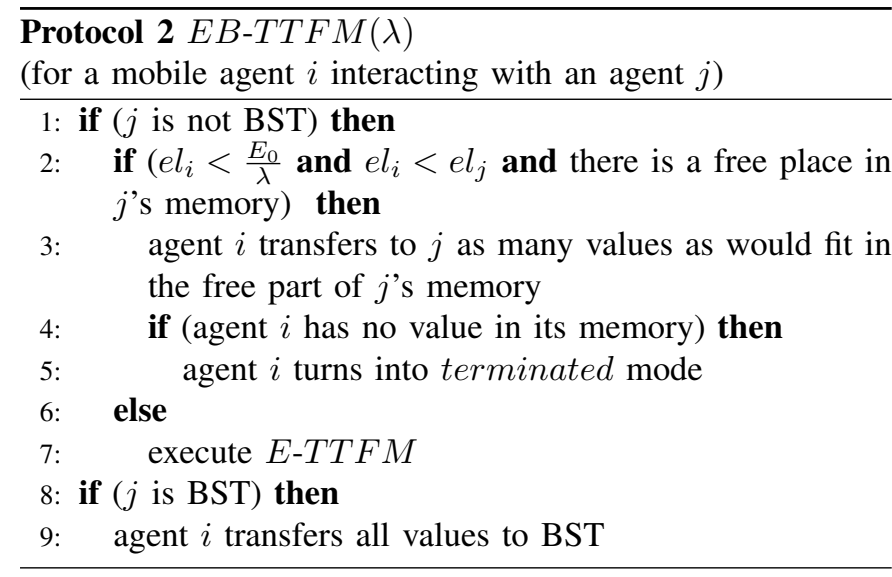

\section{A. Energy Consumption Analysis}

Intuitively, by observing the code of $E B-T T F M$, it can be seen that, if the threshold $\frac{E_{0}}{\lambda}$ is too high, an important number of interactions will result in the execution of lines $3-5$, since the condition in line 2 is satisfied easier. That concerns especially the fastest agents, since they interact more frequently. As a consequence, all the fastest agents will switch quickly to terminated mode and the values will be carried to BST by slower, even possibly slowest, agents. This will result in a worst case convergence time and energy consumption depending on $c v_{\max }$ instead of $c v_{\min }$, which is worse than for $E-T T F M$ (Prop. 1).

On the other hand, if the threshold $\frac{E_{0}}{\lambda}$ is too small, $E B$ TTFM will execute line 7 more often, i.e., behaves like $E$-TTFM, since the condition in line 2 will be difficult to satisfy. Thus, in the perspective of improving the performance of $E-T T F M$, we first study the conditions excluding the two previous (uninteresting) cases. More precisely, we study the corresponding upper and lower bounds for $E_{0}$ with respect to $\lambda$ (Lem. 1 and 2). Then, for $E_{0}$ and $\lambda$ given inside these bounds, we study the worst-case performance of EB-TTFM in terms of maximum energy spent by an agent (Th. 1). Next, we try to figure out, for a given $E_{0}$, the best choice for $\lambda$ (Sect. IV-B1), according to the results on the worst-case performance.

As explained above, the number of fastest agents turning into terminated mode during an execution plays an important role in the energy performance of EB-TTFM. Therefore, in the sequel, we classify the executions by the number of fastest agents in terminated mode at the end of the execution. Then, we study the length of the longest execution in each classification set (Prop. 2, 3 and 4), use these results to obtain the bounds for $E_{0}$ (Lem. 1 and 2) and determine the worst-case performance of EB-TTFM (Th. 1).

Definitions. We denote by $\Xi_{d}$ the set of executions of $E B$ TTFM with exactly $d$ fastest agents in terminated mode at their ends $(d \in\{0,1, \ldots,|F|\})$. For a non-empty set $\Xi_{d}$, let $m n i(d)$ be the minimum number of interactions until $d$ fastest agents are in terminated mode in the executions of $\Xi_{d}$. Let $m n v(d)$ be the minimum number of values that have been delivered to BST at the mni $(d)^{t h}$ interaction in the executions of $\Xi_{d}$ and let $\hat{e}_{d}$ be the longest execution in $\Xi_{d}$. We denote by $\theta$ the ratio between $E_{s l p}$ and $E_{w k p}\left(\theta=E_{s l p} / E_{w k p}<1\right)$.

One can see that, if $\cup_{i=1}^{|F|} \Xi_{i}=\emptyset$, no fastest agent ever turns into terminated mode and fastest agents only execute $E-T T F M$ (in line 7). In this case, EB-TTFM has the same performance as E-TTFM. On the contrary, if $\Xi_{|F|} \neq \emptyset$, there exists at least one execution, at the end of which all fastest agents are in terminated mode. Then, some values could be delivered to BST by a slow agent, increasing the convergence time of EB-TTFM (to be dependent on $c v_{\max }$ ). The case $\cup_{i=1}^{|F|} \Xi_{i}=\emptyset$ is uninteresting and the case $\Xi_{|F|} \neq \emptyset$ is undesirable. Therefore, having $\Xi_{d}$ in mind, we need to find sufficient conditions on $E_{0}$ and $\lambda$ ensuring that $\cup_{i=1}^{|F|} \Xi_{i} \neq \emptyset$ and $\Xi_{|F|}=\emptyset$.

To ensure $\cup_{i=1}^{|F|} \Xi_{i} \neq \emptyset, \operatorname{mni}(1)$ must be smaller than or equal to the length of the longest execution in $E-T T F M$ (see proof of Lem. 1). To ensure $\Xi_{|F|}=\emptyset, \operatorname{mni}(|F|)$ must be greater than the length of the longest execution in the set $\cup_{i=0}^{|F|-1} \Xi_{i}$, i.e., the set of executions converging before all the fastest agents are in terminated mode (see proof of Lem. 2). Thus, we study the values of $m n i(d)$ (Prop. 2). In addition, in order to obtain the upper bound on the length of the longest execution in Prop. 4, we compute the values of $m n v(d)$ (Prop. 3).

\section{Proposition 2: $[\operatorname{mni}(d)]$.}

A lower bound on the minimum number of interactions before $d$ fastest agents are in terminated mode, taken over all executions in a non-empty set $\Xi_{d}$ of $E B-T T F M$, is $\left\lceil\left(E_{0}-\frac{E_{0}}{\lambda}\right)\left[1-(1-\theta)^{\left\lceil\frac{d}{2}\right\rceil}\right] / E_{s l p}\right\rceil$. This bound is reached when $c v_{\min } \gg O\left(n^{2}\right)$.

Proof: Consider an execution and a fastest agent $i$. In $E B-T T F M$, fastest agent $i$ can only turn into terminated mode when the condition (in line 3) is satisfied, which means its residual energy is less than $\frac{E_{0}}{\lambda}$. During each interaction, agent $i$ cannot spend more energy than $E_{w k p}$, corresponding to the cost of the awake mode. Then the minimum number of interactions until agent $i$ turns into terminated mode, is at least $\left\lceil\frac{E_{0}-\frac{E_{0}}{\lambda}}{E_{w k p}}\right\rceil$.

As both participants to one interaction consume energy, $\operatorname{mni}(2)=\operatorname{mni}(1), \operatorname{mni}(4)=\operatorname{mni}(3), \ldots$, and more generally $\operatorname{mni}(d)=\operatorname{mni}(d-1)$ when $d$ is even. In addition, we have the following recursive equations when $d>2$ : $(m n i(d)-m n i(d-2)) \times\left(E_{w k p}-E_{s l p}\right)+m n i(d) \times E_{s l p} \geq$ $E_{0}-\frac{E_{0}}{\lambda}$. Recursively, we obtain the following relationship of $\operatorname{mni}(d)$ :

$$
\operatorname{mni}(d) \geq\left\lceil\frac{E_{0}-\frac{E_{0}}{\lambda}}{E_{s l p}}\left[1-(1-\theta)^{\left\lceil\frac{d}{2}\right\rceil}\right]\right\rceil .
$$

For the same reason in the proof of Prop. 1, when $c v_{\text {min }} \gg$ $O\left(n^{2}\right)$, the number of necessary interactions involving nonfastest agents is negligible in every $c v_{\min }$ interactions. In this 
case, the lower bound on $m n i(d)$ is reached.

Proposition 3: $[\boldsymbol{m n v}(\boldsymbol{d})]$.

When $c v_{\min } \gg O\left(n^{2}\right)$, the minimum number of values that have been delivered to BST at the $m n i(d)^{t h}$ interaction, taken over all executions in a non-empty set $\Xi_{d}$ of $E B$-TTFM, is

$$
\begin{aligned}
& m n v(d)=M(|F|-2 \beta) \times\left\lfloor\frac{m n i(d)}{2 c v_{\min }}\right\rfloor+ \\
& 2 M \times\left\lfloor\frac{m n i(1)}{2 c v_{\min }}\right\rfloor \times\left[1+\frac{\beta}{\theta}-\frac{1-\theta}{\theta^{2}}\left(1-(1-\theta)^{\beta}\right)\right],
\end{aligned}
$$

where $\beta=\lfloor(d-1) / 2\rfloor$.

Proof: It has been shown that in any segment of $2 c v_{\min }$ consecutive interactions of an execution in TTFM, at least $M|F|$ values (tight bound) are transferred to the fastest agents (Th. 11 of [6]). Similarly, it is easy to see that every $2 c v_{\min }$ interactions, at least $M|F|$ values (tight bound) are delivered to BST. However, in EB-TTFM, since the fastest agents can turn into terminated mode (in line 3), this property is no longer true. But it still holds for the segments of $2 c v_{\min }$ consecutive interactions where all fastest agents are nonterminated. According to the definition of $m n i(d)$, before the $m n i(1)^{t h}$ interaction, all fastest agents are surely nonterminated in any execution of EB-TTFM. So, we obtain at first the minimum number of of values that have been collected at the $m n i(1)^{t h}$ interaction in EB-TTFM, which is: $m n v(1)=M|F| \times\left\lfloor\frac{m n i(1)}{2 c v_{\min }}\right\rfloor$. As $m n i(1)=m n i(2)$ (Prop. $2)$, we have $m n v(1)=m n v(2)$.

Then, we consider the interval $m n i(1), m n i(3))$ of an execution. It is certain that there are at least $|F|-2$ fastest agents which are non-terminated in this interval. So we know that at least $M(|F|-2) \times\left(\left\lfloor\frac{m n i(3)}{2 c v_{\min }}\right\rfloor-\left\lfloor\frac{m n i(1)}{2 c v_{\min }}\right\rfloor\right)$ values have been delivered to BST during this interval. Recursively, we obtain the following relation between $m n v(d)$ and $m n v(d-2)$ :

$$
\begin{aligned}
& m n v(3)=m n v(4) \\
& =m n v(1)+M(|F|-2)\left(\left\lfloor\frac{m n i(3)}{2 c v_{\text {min }}}\right\rfloor-\left\lfloor\frac{m n i(1)}{2 c v_{\text {min }}}\right\rfloor\right) \\
& =M(|F|-2) \times\left\lfloor\frac{m n i(3)}{2 c v_{\min }}\right\rfloor+2 M \times\left\lfloor\frac{m n i(1)}{2 c v_{\min }}\right\rfloor ; \\
& m n v(5)=m n v(6) \\
& =m n v(3)+M(|F|-4)\left(\left\lfloor\frac{m n i(5)}{2 c v_{\min }}\right\rfloor-\left\lfloor\frac{m n i(3)}{2 c v_{\min }}\right\rfloor\right) \\
& =M(|F|-4) \times\left\lfloor\frac{m n i(5)}{2 c v_{\min }}\right\rfloor \\
& +2 M \times\left(\left\lfloor\frac{m n i(1)}{2 c v_{\text {min }}}\right\rfloor+\left\lfloor\frac{m n i(3)}{2 c v_{\text {min }}}\right\rfloor\right) ; \\
& m n v(d)=m n v(d-2)+M\left(|F|-2\left\lfloor\frac{d-1}{2}\right\rfloor\right) \\
& \times\left(\left\lfloor\frac{m n i(d)}{2 c v_{\min }}\right\rfloor-\left\lfloor\frac{m n i(d-2)}{2 c v_{\min }}\right\rfloor\right) \\
& =M\left(|F|-2\left\lfloor\frac{d-1}{2}\right\rfloor\right) \times\left\lfloor\frac{m n i(d)}{2 c v_{\min }}\right\rfloor \\
& +2 M \sum_{j=1}^{\left\lfloor\frac{d-1}{2}\right\rfloor}\left\lfloor\frac{m n i(2 j-1)}{2 c v_{\min }}\right\rfloor .
\end{aligned}
$$

Let $\beta=\left\lfloor\frac{d-1}{2}\right\rfloor$, by Prop. 2, we have, when $c v_{\min } \gg O\left(n^{2}\right)$ :

$$
\begin{gathered}
m n v(d)=M(|F|-2 \beta) \times\left\lfloor\frac{m n i(d)}{2 c v_{\min }}\right\rfloor \\
+\quad 2 M \times\left\lfloor\frac{m n i(1)}{2 c v_{\min }}\right\rfloor \times\left[1+\frac{\beta}{\theta}-\frac{1-\theta}{\theta^{2}}\left(1-(1-\theta)^{\beta}\right)\right] .
\end{gathered}
$$

Proposition 4: $\left[\left|\hat{e}_{d}\right|\right]$.

When $c v_{\min } \gg O\left(n^{2}\right)$ and $d<|F|$, the length of the longest execution $\hat{e}_{d}$ in a non-empty set $\Xi_{d}$, is at most $\rho(d)=$

$\left\lceil\frac{|N F|-2 M\left\lfloor\frac{m n i(1)}{2 c v_{\min }}\right\rfloor\left[1+\frac{1}{\theta}\left\lceil\frac{d}{2}\right\rceil-\frac{1-\theta}{\theta^{2}}\left(1-(1-\theta)^{\lceil d / 2\rceil}\right)\right]}{M(|F|-d)}\right\rceil \times 2 c v_{\text {min }}$.

Proof: The longest execution $\hat{e}_{d}$ in $\Xi_{d}$ is the one in which $d$ fastest agents are in terminated mode as fast as possible, i.e., at the $m n i(d)^{t h}$ interaction. Moreover, the data is delivered to BST in the slowest way. It means that there are $|N F|-m n v(d)$ values that have not been delivered to BST at the $m n i(d)^{t h}$ interaction of $\hat{e}_{d}$. And from the $m n i(d)^{t h}$ interaction until the termination of $\hat{e}_{d}$, only $M(|F|-d)$ values are delivered to BST during every $2 c v_{\min }$ consecutive interactions. Thus, the longest execution is of length:

$$
\left|\hat{e}_{d}\right|=m n i(d)+\left\lceil\frac{|N F|-m n v(d)}{M \times(|F|-d)}\right\rceil \times 2 c v_{\text {min }} .
$$

Therefore, when $c v_{\min } \gg O\left(n^{2}\right)$, according to Prop. 2 and Prop. 3, we have:

$$
\left|\hat{e}_{d}\right| \leq\left\lceil\frac{|N F|-2 M\left\lfloor\frac{\operatorname{mni}(1)}{2 c v_{\min }}\right\rfloor\left[1+\frac{1}{\theta}\left\lceil\frac{d}{2}\right\rceil-\frac{1-\theta}{\theta^{2}}\left(1-(1-\theta)^{\lceil d / 2\rceil}\right)\right]}{M(|F|-d)}\right\rceil \times 2 c v_{\min } .
$$

We denote the upper bound of $\left|\hat{e}_{d}\right|$ (the right hand side in the above inequality) by a function $\rho$. We can see that $\rho$ is an increasing function of $\theta$ and $d$. As $\theta=\frac{E_{s l p}}{E_{w k p}}$, when the energy spent in sleep mode increases, less interactions are needed for an agent to terminate (1). Since agents turn to terminated mode more quickly, the convergence time of EB-TTFM is longer. On the other hand, the longest execution in $\cup_{i=0}^{|F|-1} \Xi_{i}$ is at most $\rho(|F|-1)$.

Using the results above, to exclude the two previously described uninteresting cases, we compute the corresponding lower and upper bounds on the initial energy $E_{0}$ (with respect to $\lambda$ ).

Lemma 1: [Upper Bound on $E_{0}\left(\cup_{i=1}^{|F|} \Xi_{i} \neq \emptyset\right)$ ]. When $E_{0} \leq\left(2 c v_{\min }\left\lceil\frac{|N F|}{M|F|}\right\rceil-1\right) \frac{\lambda}{\lambda-1} E_{w k p}$ and $c v_{\min } \gg$ $O\left(n^{2}\right)$, there exists at least one execution of $E B-T T F M$, in which a fastest agent turns into terminated mode.

Proof: We know that the time complexity of E-TTFM is $2 c v_{\min }\left\lceil\frac{|N F|}{M|F|}\right\rceil-1$, when $c v_{\text {min }} \gg O\left(n^{2}\right)$ (Prop. 1).

To ensure that $\cup_{i=1}^{|F|} \Xi_{i} \neq \emptyset$, i.e., there exists at least one execution of $E B-T T F M$ in which a fastest agent $i$ turns into terminated mode, mni(1) must be less than or equal 
to $2 c v_{\min }\left\lceil\frac{|N F|}{M|F|}\right\rceil-1$. Elsewhere, EB-TTFM would behave as E-TTFM. Thus, we have

$$
\begin{aligned}
\operatorname{mni}(1) & \leq\left(2 c v_{\min }\left\lceil\frac{|N F|}{M|F|}\right\rceil-1\right) \frac{\operatorname{Prop} .2}{c v_{\min } \gg O\left(n^{2}\right)} \\
E_{0} & \leq\left(2 c v_{\min }\left\lceil\frac{|N F|}{M|F|}\right\rceil-1\right) \frac{\lambda}{\lambda-1} E_{w k p} .
\end{aligned}
$$

Lemma 2: [Lower Bound on $E_{0}\left(\Xi_{|F|}=\emptyset\right)$ ].

When $c v_{\text {min }} \gg O\left(n^{2}\right), \theta \leq 1 / 2$ and $E_{0} \geq$ $\left\lceil\frac{|N F|}{M \times|F|}\right\rceil \frac{\lambda}{\lambda-1} c v_{\min } E_{w k p}$, in any execution of EB-TTFM, there is at least one fastest agent, which is not in terminated mode at the end of the execution. ${ }^{5}$

Proof: We know from Prop. 4 that when $c v_{\min } \gg O\left(n^{2}\right)$ and $d<|F|$, the longest execution in $\cup_{i=0}^{|F|-1} \Xi_{i}$ is of length $\rho(|F|-1)$.

According to the definition of $m n i(d)$, the configuration, where all fastest agents are in terminated mode can happen only at or after the $\operatorname{mni}(|F|)^{t h}$ interaction in an execution. Then, if all executions in $\cup_{i=0}^{|F|-1} \Xi_{i}$ are of length less than $\operatorname{mni}(|F|)$, no execution would ever reach such a configuration, i.e., there is no execution $e$ such that all fastest agents are in terminated mode at the end of $e$. Thus, $\rho(|F|-1)<$ $\operatorname{mni}(|F|)$ implies $\Xi_{|F|}=\emptyset$.

When $c v_{\text {min }} \gg O\left(n^{2}\right)$, substituting (1) and (2) in $\rho(|F|-1)<$ $\operatorname{mni}(|F|)$, we obtain the lower bound of $E_{0}$, which is:

$$
E_{0}>\left\lceil\frac{|N F|}{M} \times \frac{\theta^{2}}{\theta^{2}+\left(\left\lceil\frac{|F|+1}{2}\right\rceil+1 / 2\right) \theta-1}\right\rceil \frac{\lambda}{\lambda-1} c v_{m i n} E_{w k p}
$$

This lower bound can be seen as a function of $\theta$, denoted by $l b(\theta)$.

Since $l b(\theta)$ is an increasing function of $\theta$ and $\theta \leq \frac{1}{2}$, we have $l b(\theta) \leq l b\left(\frac{1}{2}\right)<\left\lceil\frac{|N F|}{M} \times \frac{1}{|F|}\right\rceil \frac{\lambda}{\lambda-1} c v_{\text {min }} E_{w k p}$. So, we have a sufficient condition for $\Xi_{|F|}=\emptyset$, which is: $E_{0} \geq$ $\left\lceil\frac{|N F|}{M \times|F|}\right\rceil \frac{\lambda}{\lambda-1} c v_{\min } E_{w k p}$.

Next, we consider the worst-case performance of $E B$ TTFM with a fixed $\lambda$, supposing that every agent has an initial energy $E_{0}$ inside the bounds established above. Then from the analysis of the worst-case performance on energy consumption, we will determine the best choice for $\lambda$ in $E B$ TTFM.

Notice that if there is only one fastest agent $(|F|=1)$, the conditions of Lem. 1 and 2 are incompatible. This case is not considered by Th. 1. However, with the conditions of Lem. 2, this fastest agent stays non-terminated during all the executions and executes only $E-T T F M$. In this case, the upper bound in Prop. 1 (depending on $c v_{\text {min }}$ ) holds. Otherwise, the bound is worse, as it depends on a cover time greater than $c v_{\text {min }}$.

Theorem 1: [Worst Case Energy Analysis]. When $\left\lceil\frac{|N F|}{M \times|F|}\right\rceil \frac{\lambda}{\lambda-1} c v_{\min } E_{w k p} \leq E_{0} \leq\left(2 c v_{\min }\left\lceil\frac{|N F|}{M|F|}\right\rceil-\right.$ 1) $\lambda E_{w k p} /(\lambda-1), c v_{\text {min }} \gg O\left(n^{2}\right)$, and $|F|>1$,

$$
E s_{\max }(E B-T T F M) \leq \frac{\lambda-1}{\lambda} E_{0}+(\rho(|F|-1)-\operatorname{mni}(1)) E_{w k p},
$$

${ }^{5}$ Recall that $E_{w k p}>E_{s l p}$. For simplicity and following the study in [27], we choose $\theta \leq 1 / 2$. where $\rho(d)$ is the function defined in (2).

Proof: Consider an execution $e$ of EB-TTFM. As $E_{0} \geq$ $\left\lceil\frac{|N F|}{M \times|F|}\right\rceil \frac{\lambda}{\lambda-1} c v_{\min } E_{w k p}, \Xi_{|F|}=\emptyset$ (Lem. 2). Moreover, for a non-empty set $\Xi_{d},\left|\hat{e}_{d}\right| \leq \rho(d)$ when $c v_{\text {min }} \gg O\left(n^{2}\right)$ (Prop. 4). Therefore, since $\rho(d)$ is an increasing function of $d$, we have $|e| \leq \max _{d<|F|}\left|\hat{e}_{d}\right| \leq \rho(|F|-1)$. Let $i \in F$ be the agent which consumes the most in $e$. There are two possibilities at the end of $e$ :

1) The energy spent by agent $i$ is smaller or equal to $E_{0}-$ $\frac{E_{0}}{\lambda}$. Thus, the maximum energy spent by one agent in $e$ is smaller or equal to $\frac{\lambda-1}{\lambda} E_{0}$.

2) The energy spent by agent $i$ is greater than $E_{0}-\frac{E_{0}}{\lambda}$. Let $t_{i}$ be the number of past interactions when the residual energy of agent $i$ reaches $\frac{E_{0}}{\lambda}$. As $m n i(1)$ is the minimum number of interactions when a fastest agent turns into terminated mode (Prop. 2), we have $t_{i} \geq \operatorname{mni}(1)$. Thus, the length of the interval $\left[t_{i},|e|\right]$ is $|e|-t_{i} \leq|e|-m n i(1)$. Consequently, the maximum energy spent by one agent in $e$ is smaller or equal to $E_{0}-$ $\frac{E_{0}}{\lambda}+\left(|e|-t_{i}\right) E_{w k p} \leq \frac{\lambda-1}{\lambda} E_{0}+(|e|-m n i(1)) E_{w k p}$.

In conclusion, the maximum energy spent by one agent in the worst case of $E B-T T F M, E s_{\max }(E B-T T F M)$, is at most $\frac{\lambda-1}{\lambda} E_{0}+(\rho(|F|-1)-m n i(1)) E_{w k p}$.

A natural issue is to determine the best value for $\lambda$, that is the value that maximizes the upper bound (4) in Th. 1. This determination is not easy because of the ceiling and floor functions appearing in (4). Thus, in order to get an exploitable expression, we drop, in the next subsection, the assumption of bounded memory and we consider that an agent can hold an arbitrary number of values. Then we obtain a better upper bound using such a $\lambda$.

\section{B. Special Case of Non-Bounded Memory $(M \geq n-1)$}

The following improved bound for $E_{0}$ (with respect to Lem. 2) is obtained when the lower bound of $\operatorname{mni}(|F|)$ is larger or equal to $c v_{\min }$. This condition ensures that at least one fastest agent is not terminated during the first $c v_{\text {min }}$ interactions (and not until convergence as in Lem. 2). During this period, the fastest agents meet all the non-fastest ones, collect all their values (as $M$ is large enough), and turn them into terminated mode, as it is stated by Lem. 3. This certainly ensures that $\Xi_{|F|}=\emptyset$.

Lemma 3: [Lower Bound on $E_{0}$ when $M \geq n-1\left(\Xi_{|F|}=\right.$ $\emptyset)]$. When $E_{0} \geq \frac{\lambda}{\lambda-1}\left(\frac{\theta}{1-(1-\theta)^{\left\lceil\frac{|F|}{2}\right\rceil}}\right) c v_{\min } E_{w k p}$ and $M \geq$ $n-1$, in any execution of EB-TTFM, all non-fastest agents are in terminated mode after the first $c v_{\min }$ interactions.

Proof: We know that the state where all fastest agents are in terminated mode can happen only at or after the $\operatorname{mni}(|F|)^{t h}$ interaction. Thus, if $\operatorname{mni}(|F|) \geq c v_{\text {min }}$, in any execution of EB-TTFM, there is at least one nonterminated fastest agent at the end of the first $c v_{\min }$. Moreover, all the non-fastest mobile agents are in terminated mode, since every non-fastest mobile agent interacts with a 
non-terminated fastest agent during the first $c v_{\min }$ and has transferred all of its value (as the memory is unbounded) in this interval.

Substituting $\operatorname{mni}(|F|)$ by the lower bound in (1) and expressing the formula in function of $E_{0}$ yields the formula appearing in Lemma 3.

Then, similarly to the bounded case in the previous section, we compute $E s_{\max }(E B-T T F M)$ for $E_{0}$ satisfying the bounds of Lemmas 1 and 3 .

Theorem 2: [Worst Case Energy Analysis when $M \geq n-1]$. When $\frac{\lambda}{\lambda-1}\left(\frac{\theta}{1-(1-\theta)^{\left\lceil\frac{|F|}{2}\right\rceil}}\right) c v_{\min } E_{w k p} \leq E_{0} \leq$ $\left(2 c v_{\min }-1\right) \frac{\lambda}{\lambda-1} E_{w k p}, E s_{\max }(E B-T T F M) \leq \frac{\lambda-1}{\lambda} E_{0}+$ $\left(1+\frac{1}{2-\theta}\right) c v_{\min } E_{w k p}=\Theta\left(c v_{\min } E_{w k p}\right)$

Proof: Assume that for an execution $e$, the energy of agent $i$ decreases to $\frac{E_{0}}{\lambda}$ at time $t_{i}$. Then, there are two possible states for agent $i$ after $t_{i}+c v_{\text {min }}$ :

- Agent $i$ is in terminated mode: It means that agent $i$ has already transferred its value. Therefore, in this case, the residual energy of agent $i$ at the end of execution, $e l_{i}(|e|)=e l_{i}\left(t_{i}+c v_{\min }\right) \geq \frac{E_{0}}{\lambda}-c v_{\min } E_{w k p}$.

- Agent $i$ is not in terminated mode:

If agent $i$ is still alive after $t_{i}+c v_{\text {min }}$, for any interaction $(i, j)$ at time $t \in\left[t_{i}, t_{i}+c v_{\text {min }}\right]$ with an agent $j$ still nonterminated, $e l_{j}(t)<e l_{i}(t) \leq \frac{E_{0}}{\lambda}$. Thus, after $t_{i}+c v_{\text {min }}$, agent $i$ is the only non-terminated agent. And agent $i$ is the agent that transfers the last value to the base station. Let agent $j$ be the last agent turning into terminated mode during $\left[t_{i}, t_{i}+c v_{\text {min }}\right]$ and let $t_{j}$ be the time where its energy decreases to $\frac{E_{0}}{\lambda}$. Let $t_{i j} \in\left[t_{i}, t_{i}+c v_{\text {min }}\right]$ be the time when non-terminated $j$ interacts with $i$. We know that $t_{i j}>t_{j}$ since $e l_{j}\left(t_{i j}\right)<e l_{j}\left(t_{j}\right)=\frac{E_{0}}{\lambda}$. Denoting by $x_{i}$ the number of interactions of agent $i$ during $\left[t_{i}, t_{i j}\right]$ and by $x_{j}$ the number of interactions of agent $j$ during $\left[t_{j}, t_{i j}\right]$ :

1) First, according to the definition of $x_{i}$ and $x_{j}$, we have $x_{i} \leq t_{i j}-t_{i}, x_{j} \leq t_{i j}-t_{j}$.

2) As $e l_{j}\left(t_{i j}\right)<e l_{i}\left(t_{i j}\right), \frac{E_{0}}{\lambda}-x_{j}\left(E_{w k p}-E_{s l p}\right)-\left(t_{i j}-\right.$ $\left.t_{j}\right) E_{s l p}<\frac{E_{0}}{\lambda}-x_{i}\left(E_{w k p}-E_{s l p}\right)-\left(t_{i j}-t_{i}\right) E_{s l p}$. Thus, we have $x_{i}<x_{j}+\left(t_{i}-t_{j}\right) \frac{\theta}{1-\theta}$ where $\theta=$ $\frac{E_{s l p}}{E_{w k p}}$

3) There is no interaction between $i$ and $j$ during the interval $\left[\min \left(t_{i}, t_{j}\right), t_{i j}\right)$. Indeed, suppose that there is one at time $t^{\prime} \in\left[\min \left(t_{i}, t_{j}\right), t_{i j}\right)$, $k_{1}=\arg \min _{x \in\{i, j\}} t_{x}$ and $k_{2}=\{i, j\} \backslash k_{1}$. We know that $e l_{k_{1}}\left(t^{\prime}\right) \leq e l_{k_{1}}\left(t_{k_{1}}\right)=\frac{E_{0}}{\lambda}$. If $e l_{k_{1}}\left(t^{\prime}\right)<$ $e l_{k_{2}}\left(t^{\prime}\right)$, agent $k_{1}$ transfers its values to agent $k_{2}$. If $e l_{k_{1}}\left(t^{\prime}\right)>e l_{k_{2}}\left(t^{\prime}\right)$, as $e l_{k_{2}}\left(t^{\prime}\right)<e l_{k_{1}}\left(t^{\prime}\right) \leq \frac{E_{0}}{\lambda}$, agent $k_{2}$ transfers its values to agent $k_{1}$. Then, after the interaction at time $t^{\prime}$, either agent $k_{1}$ or agent $k_{2}$ is in terminated mode which contradicts the interaction of $(i, j)$ at time $t_{i j}$ where both agents are non-terminated.

Since there is no interaction between agent $i$ and $j$ during the interval $\left[\min \left\{t_{i}, t_{j}\right\}, t_{i j}\right)$ and since there is at least one interaction between $i$ and $j$ for each $c v_{\text {min }}$ time interval, according to the definition of a cover time, we have $t_{i j} \leq \min \left\{t_{i}, t_{j}\right\}+c v_{\text {min }}$. Thus, $x_{i}+x_{j} \leq c v_{\text {min }}$.

Taken all the inequalities obtained above into consideration, $x_{i}$ reaches its maximum value $\frac{1}{2-\theta} c v_{\min }$ when $t_{i j}-t_{j}=c v_{\text {min }}, t_{i j}-t_{i}=\frac{1}{2-\theta} c v_{\text {min }}, x_{j}=\frac{1-\theta}{2-\theta} c v_{\text {min }}$. Figure 1 gives an illustration of this case. As the ex-

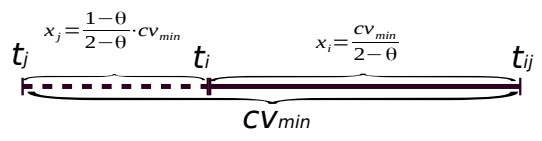

Fig. 1. Illustration of the case where $x_{i}$ has the maximum value.

ecution ends before $t_{i j}+c v_{\text {min }}$, agent $i$ interacts at most $c v_{\text {min }}$ times after $t_{i j}$. Thus, from $t_{i}$ to the end of execution, agent $i$ interacts at most $x_{i}+c v_{\min }=\frac{3-\theta}{2-\theta}$ times. Therefore, in the worst case, agent $i$ will consume $\frac{3-\theta}{2-\theta} c v_{\min } E_{w k p}$ energy at the end of execution $e$.

In conclusion, for any agent $i \in F$ whose energy decreases to $\frac{E_{0}}{\lambda}$ before the end of an execution $e$, we have proved that agent $i$ should consume at most $\frac{3-\theta}{2-\theta} c v_{\min } E_{w k p}$ energy. Therefore, we have $E s_{\max }(E B-T T F M) \leq \frac{\lambda-1}{\lambda} E_{0}+(1+$ $\left.\frac{1}{2-\theta}\right) c v_{\min } E_{w k p}$.

1) Best Choice for $\lambda$ : Now, given $E_{0}$ (large enough for accomplishing the task), we study the best choice for $\lambda$ for minimizing $E s_{\max }(E B-T T F M)$. We can see from Th. 2 that $E s_{\max }(E B-T T F M)$ decreases when $\lambda$ decreases. But $\lambda$ should satisfy the condition $E_{0} \geq \frac{\lambda}{\lambda-1}(\theta /(1-(1-$ $\left.\theta)^{\left\lceil\frac{|F|}{2}\right\rceil}\right) c v_{\min } E_{w k p}$. Therefore, the smallest value of $\lambda$ is obtained when $\frac{\lambda}{\lambda-1}\left(\frac{\theta}{1-(1-\theta)^{\left\lceil\frac{|F|}{2}\right\rceil}}\right) c v_{\min } E_{w k p}=E_{0}$, which is $\tilde{\lambda}=E_{0} /\left(E_{0}-\left(\frac{\theta^{1-(1-\theta)}}{1-(1-\theta)^{\left\lceil\frac{|F|}{2}\right\rceil}}\right) c v_{\min } E_{w k p}\right)$

2) Interpretation of Results: Comparison of Energy Performance between EB-TTFM and E-TTFM: We give a meaningful example of the interest to have analytical functions for describing the energy consumption performance. This example considers the unbounded memory case and illustrates conditions under which EB-TTFM outperforms E-TTFM. When the memory is not bounded and $c v_{\min } \gg O\left(n^{2}\right)$, by Prop. 1, Es $s_{\max }(E-T T F M)=\left(2 c v_{\min }-1\right) E_{w k p}$, and by Th. 2 , when $\lambda$ is set to $\tilde{\lambda}, E s_{\max }(\operatorname{EB}-T T F M(\tilde{\lambda})) \leq$ $\left(1+\theta /\left(1-(1-\theta)^{\left\lceil\frac{|F|}{2}\right\rceil}\right)+\frac{1}{2-\theta}\right) c v_{\min } E_{w k p}$. Then, we obtain that for $|F| \geq 10$ and $\theta \leq(3-\sqrt{5}) / 2 \approx 0.38$, $E s_{\max }(E B-T T F M(\tilde{\lambda})) \leq E s_{\max }(E-T T F M)$, i.e., $E B-$ TTFM $(\tilde{\lambda})$ outperforms E-TTFM.

The figure below represents the comparison of $E s_{\max }$ between $\operatorname{EB}-T T F M(\tilde{\lambda})$ and $E-T T F M$ with different $|F|$ and $\theta$. The red (lighter) plane shows $E s_{\max }(E-T T F M)$ and 
the blue (darker) one represents $E s_{\max }(E B-T T F M(\tilde{\lambda}))$. We can see that $E B-T T F M(\tilde{\lambda})$ is more energy balanced when $\theta$ is small, i.e., when the energy spent in sleep mode is much less than the energy spent in awake mode.

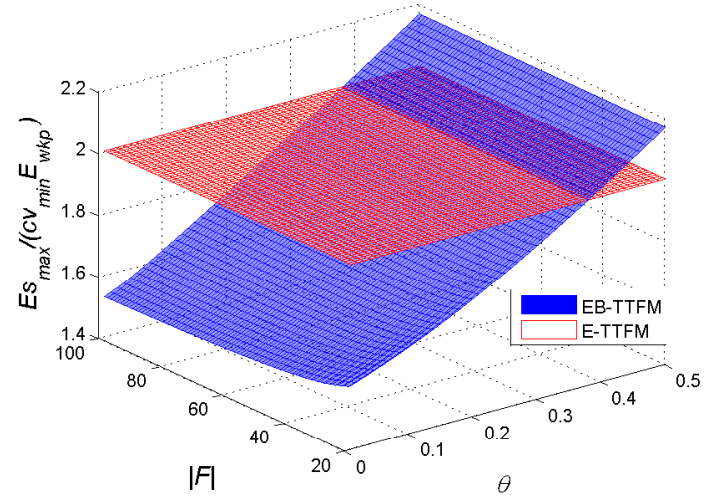

3) Interpretation of Results: Relationship between $\lambda, E_{0}$ and $E s_{\max }$ in EB-TTFM.: In the Section IV-B1, we discussed the best choice for $\lambda$ in EB-TTFM. But what if the value of $\lambda$ is not set to $\tilde{\lambda}$ (Eq. IV-B1) and what is the performance of $E B-T T F M$ in this case? In this section, we use $3 \mathrm{D}$ plots to show the relation among $\lambda, E_{0}$ and $E s_{\max }(E B-T T F M)$.

Firstly, from Lemma 3 , we have a lower bound for $E_{0}$ depending on $\lambda$. Then, 3D plots of $\lambda, E_{0}$ and $E s_{\max }$ can be constructed following Th. 2. Figure 2 shows the performance of $E B$-TTFM where $|F|=10, \theta=0.2$. $\mathrm{x}$ axis presents $\lambda$, $\mathrm{y}$ axis shows $E_{0}$ and $\mathrm{z}$ axis shows $E s_{\max }(E B-T T F M)$.

The blue polygon in Figure 2a presents the possible values of $\lambda$ and $E_{0}$ for which EB-TTFM does not behave like $E-T T F M$. As we can see, $\lambda$ should be carefully chosen to a small value for $E B-T T F M$ to perform better than $E-T T F M$ in the worst case. The green border on the blue polygon represents the best choice for $\lambda$ (Sect. IV-B1). The red polygon in Figure 2a shows the region of values for $\lambda$ and $E_{0}$ where EB-TTFM behaves like E-TTFM.

Figure $2 \mathrm{~b}$ gives a view of $x, y$ axis which shows the feasible values of $\lambda$ and $E_{0}$ (blue and red area).

(a):

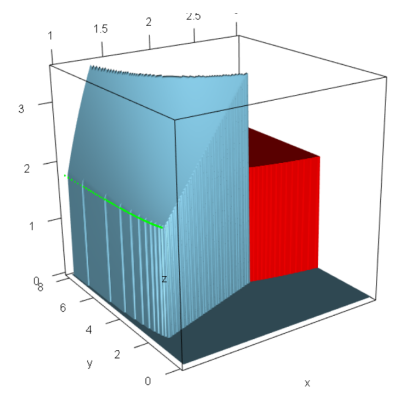

(b):

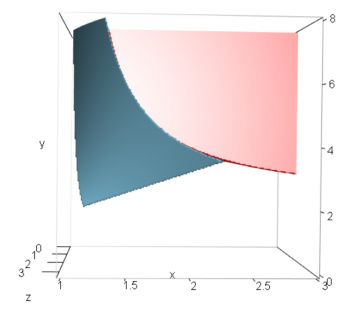

Fig. 2. 3D plot on the relationship of $\lambda, E_{0}$ and $E s_{\max }$

\section{LOWER BOUND ON $E s_{\max }$}

In this section, we present a lower bound (Th. 3) on the maximum energy spent by an agent $\left(E s_{\max }\right)$ for achieving data collection. The protocols considered here, like in the whole study, are those that can compare (but not use in any other way) the cover times and the residual energies of interacting agents. Let us denote this class of protocols by $\mathbf{P}^{c v \cup e}$. Following the intuition above for strategies of energybalanced data collection protocols, we study the lower bound for different sub-classes of protocols in $\mathbf{P}^{c v \cup e}$, distinct in their strategies of turning agents into terminated mode.

Hence, we decompose $\mathbf{P}^{c v \cup e}$ into subsets $\mathbf{P}_{i}$, defined below. For that, we order the agents according to their cover times and denote by $n s$ the number of different cover times and by $F_{i}$ the set of mobile agents with the the $i^{t h}$ smallest cover time. Thus the set of fastest agents is $F_{1}$. We also denote by $c v^{i}$ the value of the $i^{t h}$ smallest cover time. Then, we define $\mathbf{P}_{i}$ as the class of protocols for which, there is at least one non-terminated agent in $\cup_{j=1}^{i} F_{j}$ at the end of any execution (or turning into terminated mode during the last interaction of the execution). Moreover, in $\mathbf{P}_{i}$, when $i>1$, there exists at least one execution in which all agents in $\cup_{j=1}^{i-1} F_{j}$ are terminated before the end of execution, i.e.,

$$
\begin{aligned}
\mathbf{P}_{i} & =\left\{\mathcal{P} \in \mathbf{P}^{c v \cup e} \mid\right. \\
& \forall \quad e \in \mathbf{e}(\mathcal{P}), \exists f \in \cup_{j=1}^{i} F_{j}, \operatorname{Mode}(f, e) \neq \text { terminated } \wedge \\
& \text { if } \quad(i>1): \\
& \left.\exists \quad e \in \mathbf{e}(\mathcal{P}), \forall f \in \cup_{j=1}^{i-1} F_{j}, \operatorname{Mode}(f, e)=\text { terminated }\right\},
\end{aligned}
$$

where $\mathbf{e}(\mathcal{P})$ is the set of all executions in protocol $\mathcal{P}$ and Mode $(f, e) \in\{$ awake, sleep, terminated $\}$ indicates the energy consumption mode of agent $f$ just before the last interaction in execution $e$.

By definition, the $\mathbf{P}_{i}$ 's are disjoint and $\cup_{i=1}^{n s} \mathbf{P}_{i}=\mathbf{P}^{c v \cup e}$. Moreover, E-TTFM belongs to $\mathbf{P}_{1}$. Note that EB-TTFM can belong to different subsets depending on the value of $\frac{E_{0}}{\lambda}$. Under the conditions of Lem. 2, EB-TTFM is in $\mathbf{P}_{1}$.

We obtain the lower bound on energy using in particular the lower bound on time (the length of an execution) of [6]. This bound holds for any data collection protocol using only cover time comparisons. Let $\mathbf{P}^{c v}$ be this class of protocols. The proof of the lower bound on time for $\mathbf{P}^{c v}$ in [6] holds also for $\mathbf{P}^{c v \cup e}$, because only constraints related to the data collection problem are invoked. For example, it is necessary that every mobile agent either meets BST by itself, or meets at least one other agent that meets BST, before convergence. Then comparisons of the residual energies of two interacting agents cannot produce a shorter execution. This is expressed in the following observation.

Observation: The lower bound on time for any data collection in $\mathbf{P}^{c v}[6]$ is also correct for any protocol in $\mathbf{P}^{c v \cup e}$.

Recall that this bound is tight and equal to the upper bound on time of the protocol TTFM, also presented in [6]. Hence, we denote this bound here by $\left|e_{\max }(T T F M)\right|$.

The lower bound on energy presented below holds for the large and natural family of cover time vectors for which $c v_{\text {min }} \gg O\left(n^{2}\right)$ (see justifications in Sect. II-A). 
Lemma 4: [Lower Bound on $E s_{\max }$ for Protocols in $\mathbf{P}_{i}$ ] If $c v_{\text {min }} \gg O\left(n^{2}\right)$, for any protocol $\mathcal{P}$ in $\mathbf{P}_{i}$, the energy spent by an agent in the worst case, is at least $E s_{\max }(E-T T F M) /\left\lceil\frac{\left|\cup_{j=1}^{i} F_{j}\right|}{2}\right\rceil$.

Proof: First, consider the following schedule which repeats the segment $X_{1} X_{2}$, where $X_{1} X_{2}$ is of length $c v_{\text {min }}$. $X_{1} \equiv[$ repeat as much as possible all the possible interactions between agents in $\cup_{j=1}^{i} F_{j}$ ],

$X_{2} \equiv[$ all the other necessary interactions to satisfy $c v \mathrm{~s}]$.

Consider now the execution of $\mathcal{P} \in \mathbf{P}_{i}$ for this schedule, denoted by $e$. Since no agent consumes more than $E s_{\max }(\mathcal{P})$ amount of energy in any execution of $\mathcal{P}$, when an agent consumes $E s_{\max }(\mathcal{P})$ in $e$, either it turns into terminated mode or $e$ reaches the final configuration. And as $\mathcal{P} \in \mathbf{P}_{i}$, there is at least one agent in $\cup_{j=1}^{i} F_{j}$ which is non-terminated at the end of $e$. Moreover, notice that $\left|X_{2}\right| \leq \frac{n(n-1)}{2}=O\left(n^{2}\right)$ and as $c v_{\min } \gg O\left(n^{2}\right)$, the length of $X_{2}$ is negligible. Thus, following the above schedule, at time $t=\frac{E s_{\max }(\mathcal{P})}{E_{w k p}} \times\left\lceil\frac{\left|\cup_{j=1}^{i} F_{j}\right|}{2}\right\rceil$, all agents in $\cup_{j=1}^{i} F_{j}$ consume $E s_{\max }(\mathcal{P})$ amount of energy. However, the length of $e$ must be smaller than or equal to $t$. Because otherwise, either there would be a non-terminated agent consuming more than $E s_{\max }$, or all agents would be terminated, which contradicts the fact that $\mathcal{P} \in \mathbf{P}_{i}$. Thus, we have $t \geq|e|$, i.e., $\frac{E s_{\max }(\mathcal{P})}{E_{w k p}} \times\left\lceil\frac{\left|\cup_{j=1}^{i} F_{j}\right|}{2}\right\rceil \geq|e|$.

By the observation above, execution $e$ satisfies the lower bound on time of [6]. Thus, we obtain $|e| \geq$ $\left|e_{\max }(T T F M)\right|=\left|e_{\max }(E-T T F M)\right|$, as $E-T T F M$ has the same time performance as TTFM (Sect. III). Moreover, by Prop. 1, when $c v_{\min } \gg O\left(n^{2}\right),\left|e_{\max }(E-T T F M)\right| E_{w k p}=$ $E s_{\max }(E-T T F M)$. Then we have

$$
\begin{aligned}
E s_{\max }(\mathcal{P}) & \geq \frac{|e| \times E_{w k p}}{\left\lceil\frac{\left|\cup_{j=1}^{i} F_{j}\right|}{2}\right\rceil} \geq \frac{\left|e_{\max }(E-T T F M)\right| E_{w k p}}{\left\lceil\frac{\left|\cup_{j=1}^{i} F_{j}\right|}{2}\right\rceil} \\
& =E s_{\max }(E-T T F M) /\left\lceil\frac{\left|\cup_{j=1}^{i} F_{j}\right|}{2}\right\rceil .
\end{aligned}
$$

Lemma 5: [Lower Bound on $E s_{\max }$ for Protocols in $\mathbf{P}_{i}$ ] If $c v_{\text {min }} \gg O\left(n^{2}\right)$, for any protocol $\mathcal{P}$ in $\mathbf{P}_{i}$, the energy spent by an agent in the worst case, is at least $c v^{i} E_{w k p}$.

Proof: When $i>1$, as $\mathcal{P} \in \mathbf{P}_{i}$, there exists at least one execution $e$ in which all agents in $\cup_{j=1}^{i-1} F_{j}$ are terminated before the end of the execution. Let $t$ indicate the time when all agents in $\cup_{j=1}^{i-1} F_{j}$ are terminated in $e$ and let $f \notin \cup_{j=1}^{i-1} F_{j}$ be a non-terminated agent (with cover time $c v_{f} \geq c v^{i}$ ), holding one of the values not yet collected by BST at time $t+1$. We prove the existence of another execution $e^{\prime}$ such that $E s_{\max }\left(e^{\prime}\right) \geq c v^{i} E_{w k p}$. The execution $e^{\prime}$ begins with the same prefix of $t$ events as $e$ and continues by meetings between agent $f$ and the terminated agents until $f$ meets BST at time $t+c v_{f}$. In our energy consumption scheme, when a sleeping agent meets a terminated agent, it consumes $E_{w k p}$ and does not change its mode (See Sect. II-B). Therefore and because $c v_{f}>c v_{\min } \gg O\left(n^{2}\right)$, during the interval $\left[t, t+c v_{f}\right]$, agent $f$ consumes $c v_{f} E_{w k p}$ (the necessary interactions involving other agents than $f$ during $c v_{f}$ are negligible, as in the proof of Lem. 4). So, when $i>1$, we have $E s_{\max }(\mathcal{P}) \geq E s_{\max }\left(e^{\prime}\right) \geq$ $c v_{f} E_{w k p} \geq c v^{i} E_{w k p}$.

Next, we consider the case where $i=1$. We prove that $E s_{\max }(\mathcal{P}) \geq c v_{\min } E_{w k p}$ by contradiction. Suppose that there exists a protocol $\mathcal{P} \in \mathbf{P}_{1}$ such that $E s_{\max }(\mathcal{P})<$ $c v_{\min } E_{w k p}$. To contradict this, we construct an execution $e$ of $\mathcal{P}$ with $E s_{\max }(e) \geq c v_{\min } E_{w k p}$. Consider again the schedule $X_{1} X_{2}$ in the proof of Lem. 4. It is possible that at time $t=E s_{\max }(\mathcal{P}) / E_{w k p}<c v_{\min }$, an agent $j \in F_{1}$ consumes $E s_{\max }(\mathcal{P})$ and turns into terminated. For the same reason as above, we can construct $e$ in which a non-terminated agent $f$ meets the terminated agent $j$ repetitively until it meets others at time $t+c v_{f}$. Thus, when $i=1$, we have $E s_{\max }(\mathcal{P}) \geq c v_{\min } E_{w k p}$.

Theorem 3: [Lower Bound on $E s_{\max }$ for Protocols in $\mathbf{P}^{c v \cup e}$ ] If $c v_{\min } \gg O\left(n^{2}\right)$, for any protocol in $\mathbf{P}^{c v \cup e}$, the energy spent by an agent in the worst case is at least $\max \left\{E s_{\max }(E-T T F M) /\left\lceil\frac{|F|}{2}\right\rceil, c v_{\min } E_{w k p}\right\}$.

Proof: The result comes directly from Lem. 4 and 5, since $\cup_{i} \mathbf{P}_{i}=\mathbf{P}^{c v \cup e}$ and

$$
\begin{aligned}
& \min _{i} \max \left\{E s_{\max }(E-T T F M) /\left\lceil\frac{\left|\cup_{j=1}^{i} F_{j}\right|}{2}\right\rceil, c v^{i} E_{w k p}\right\} \\
= & \max \left\{E s_{\max }(E-T T F M) /\left\lceil\frac{|F|}{2}\right\rceil, c v_{\min } E_{w k p}\right\} .
\end{aligned}
$$

Next, we identify the cases where this bound is reached by the presented protocols (Cor. 1), and where it differs only by a constant multiplicative factor from the energy complexity of $E B-T T F M$, for the case of non-bounded memory and with a good choice of $\lambda$ (Cor. 2).

Corollary 1: When $|F| \leq 2$ and $c v_{\min } \gg O\left(n^{2}\right), E$ TTFM is energy-optimal in $\mathbf{P}^{c v \cup e}$, with respect to the energy spent by an agent in the worst case.

Proof: From Th. 3, if $|F| \leq 2, \forall \mathcal{P} \in$ $\mathbf{P}^{c v \cup e}, E s_{\max }(\mathcal{P}) \geq E s_{\max }(E-T T F M)$. As $E$ $T T F M \in \mathbf{P}^{c v \cup e}, E-T T F M$ is energy optimal in $\mathbf{P}^{c v \cup e}$.

Corollary 2: When $|F|>2$ and $c v_{\min } \gg O\left(n^{2}\right)$, for any protocol in $\mathbf{P}^{c v \cup e}$, the energy spent by an agent in the worst case is at least $c v_{\min } E_{w k p}$. For the case where $M \geq n-1$ and $\lambda$ satisfies the conditions of Th. 2, EB-TTFM( $\lambda)$ reaches this bound asymptotically.

Proof: This result comes directly from Th. 3 and Th. 2.

\section{CONCLUSION}

This paper presents a formal model for power-aware distributed algorithms. Designing a good model is difficult. If the model is too abstract (or general), it won't easily apply to reality, if it is too low level and considers too many parameters, it won't allow formal treatments. We believe that the model we introduced is a good compromise between the two approaches. 
On the one hand, it is based on practical information on how real resource-limited mobile sensor networks are functioning, and on the other hand, as shown in the paper, it allows to obtain analytically precise formulas, which are obviously not directly intuitive.

Moreover, the proposed model is adaptable to other types of mobile sensor networks and their applications (e.g., to the case where the transmitted data does not always fit into one packet). As already noticed, the assumption on the cover times does not particularize the model, since almost all real mobile sensor networks either operate in a bounded area (town, factory, security zone, etc.) or satisfy the home coming tendency (networks related to human or animal mobility). Nevertheless, other kinds of "periodic" conditions on the interactions (e.g., probabilistic interactions) can be considered to study energy consumption using the proposed framework. Finally, other important communication problems (as broadcast, all-to-all communication, routing, etc.) have many points in common with data collection (excepted that unique identifiers may be needed, like in community protocols [31]). Hence, the techniques developed here can be useful in future studies of these problems.

The second contribution of the paper consists in establishing analytical formulas related to the energy consumption of two data collection algorithms. We want to emphasize that having analytical formulas allows obtaining information that could not be obtained by the sole intuition, and that would be difficult to obtain by simulations. On one hand, the study of the curves and plots allows to get easily the conditions on the parameters (e.g., think of the value of $\frac{3-\sqrt{5}}{2}$ for $\theta$ in Sect. IV-B2). On the other hand, the analytical approach allows obtaining general upper and (always difficult to establish) lower bounds.

To conclude, we summarize the main technical results of the paper. In Sect. IV-A, we obtain formulas for the worstcase performance of energy in EB-TTFM. Then, for the case of non-bounded memory, we compute the best value for $\lambda$ with respect to $E_{0},|F|, E_{w k p}, E_{s l p}$ and $c v_{\min }$ (Sect. IV-B). Further analysis with the best $\lambda$ shows that no matter how much initial energy an agent has, once it consumes more than some calculated amount $E_{f i x}$, the best strategy is to switch to terminated mode, when interacting with a more powerful agent. We interpret the formulas graphically, and show that EB-TTFM is more energy efficient in the case where the number of fastest agents are more than 10 and the ratio between $E_{s l p}$ and $E_{w k p}$ is less then $\frac{3-\sqrt{5}}{2}$ (Sect. IV-B2). At the end (Sect. V), we give a uniform lower bound for energy consumption for all possible data collection protocols. We identify the cases where this bound is reached by the presented protocols (Cor. 1), and where it differs only by a constant multiplicative factor from the energy upper bound of EB-TTFM (Cor. 2).

\section{REFERENCES}

[1] P. Juang, H. Oki, Y. Wang, M. Martonosi, L. S. Peh, and D. Rubenstein, "Energy-efficient computing for wildlife tracking: Design tradeoffs and early experiences with zebranet," ACM Sigplan Notices, vol. 37, no. 10, pp. 96-107, 2002.
[2] D. Angluin, J. Aspnes, Z. Diamadi, M. J. Fischer, and R. Peralta, "Computation in networks of passively mobile finite-state sensors," Distributed computing, vol. 18, no. 4, pp. 235-253, 2006.

[3] R. Fish, M. Flickinger, and J. Lepreau, "Mobile emulab: A robotic wireless and sensor network testbed," in IEEE INFOCOM, 2006.

[4] J. Polastre, J. Hill, and D. Culler, "Versatile low power media access for wireless sensor networks," in Embedded networked sensor systems, ACM. ACM, 2004, pp. 95-107.

[5] A. Boulmaiz, D. Messadeg, N. Doghmane, and A. Taleb-Ahmed, "Robust acoustic bird recognition for habitat monitoring with wireless sensor networks," I. J. Speech Technology, vol. 19, no. 3, pp. 631-645, 2016.

[6] J. Beauquier, J. Burman, J. Clement, and S. Kutten, "On utilizing speed in networks of mobile agents," in PODC. ACM, 2010, pp. 305-314.

[7] C. Dwork, N. A. Lynch, and L. J. Stockmeyer, "Consensus in the presence of partial synchrony," J. ACM, vol. 35, no. 2, pp. 288-323, 1988.

[8] M. K. Aguilera and S. Toueg, "Timeliness-based wait-freedom: a gracefully degrading progress condition," in $P O D C, 2008$, pp. 305-314.

[9] O. Michail and P. G. Spirakis, "Terminating population protocols via some minimal global knowledge assumptions," J. Parallel Distrib. Comput., vol. 81-82, pp. 1-10, 2015.

[10] M. McNett and G. M. Voelker, "Access and mobility of wireless PDA users," Mobile Computing and Communications Review, vol. 9, no. 2, pp. $40-55,2005$.

[11] S. Hong, I. Rhee, S. J. Kim, K. Lee, and S. Chong, "Routing performance analysis of human-driven delay tolerant networks using the truncated levy walk model," in Mobility Models, 2008, pp. 25-32.

[12] T. Karagiannis, J. L. Boudec, and M. Vojnovic, "Power law and exponential decay of inter contact times between mobile devices," in MOBICOM, 2007, pp. 183-194.

[13] H. Cai and D. Y. Eun, "Crossing over the bounded domain: from exponential to power-law inter-meeting time in MANET," in MOBICOM, 2007, pp. 159-170.

[14] "The Dartmouth wireless trace archive http://crawdad.cs.dartmouth.edu/." Dartmouth College, 2007.

[15] I. Rhee, M. Shin, S. Hong, K. Lee, and S. Chong, "On the levy-walk nature of human mobility," in INFOCOM, 2008, pp. 924-932.

[16] B. Raffaele, B. Roberto, D. Franco, and C. Flavio, "Energy efficiency in the future internet: a survey of existing approaches and trends in energy-aware fixed network infrastructures," Communications Surveys \& Tutorials, IEEE, vol. 13, no. 2, pp. 223-244, 2011.

[17] T. Sunil and K. Ashwani, "A survey of routing protocols in mobile ad hoc networks," International Journal of Innovation, Management and Technology, vol. 1, no. 3, p. 279, 2010.

[18] M. Di Francesco, S. K. Das, and G. Anastasi, "Data collection in wireless sensor networks with mobile elements: A survey," ACM TOSN, vol. 8, no. 1, p. 7, 2011.

[19] P. Luciana, P. Andrea, and C. Marco, "Opportunistic networking: data forwarding in disconnected mobile ad hoc networks," Communications Magazine, IEEE, vol. 44, no. 11, pp. 134-141, 2006.

[20] S. E. Nikoletseas, T. P. Raptis, and C. Raptopoulos, "Interactive wireless charging for energy balance," in ICDCS 2016, Nara, Japan, June 27-30, 2016, 2016, pp. 262-270.

[21] Y. Wu, X. Y.Li, Y. H.Liu, and W.Lou, "Energy-efficient wake-up scheduling for data collection and aggregation," IEEE Transactions on Parallel and Distributed Systems., vol. 21, no. 2, pp. 275-287, 2010.

[22] T. Herman, S. V. Pemmaraju, L. Pilard, and M. Mjelde, "Temporal partition in sensor networks," in SSS, 2007, pp. 325-339.

[23] Y. Yao, Q. Cao, and A. V. Vasilakos, "Edal: An energy-efficient, delayaware, and lifetime-balancing data collection protocol for heterogeneous wireless sensor networks," IEEE/ACM Transactions on Networking., vol. 23 , no. 3, pp. 810-823, 2015.

[24] E. J. C. S. Bandyopadhyay, "An energy efficient hierarchical clustering algorithm for wireless sensor networks," in INFOCOM, vol. 3. IEEE, 2003, pp. 1713-1723.

[25] R. Wattenhofer, L. Li, P. Bahl, and Y. M. Wang, "Distributed topology control for power efficient operation in multihop wireless ad hoc networks," in INFOCOM, vol. 3. IEEE, 2001, pp. 1388-1397.

[26] Y. Choi, G. Pandurangan, M. Khan, and V. S. A. Kumar, "Energyoptimal distributed algorithms for minimum spanning trees," IEEE Journal on Selected Areas in Communications, vol. 27, no. 7, pp. 1297 1304, 2009. 
[27] V. Rajendran, K. Obraczka, and J. J. Garcia-Luna-Aceves, "Energyefficient, collision-free medium access control for wireless sensor networks," Wireless Networks, vol. 12, no. 1, pp. 63-78, 2006.

[28] H. Tan and I. Korpeoglu, "Power efficient data gathering and aggregation in wireless sensor networks," ACM Sigmod Record, vol. 32, no. 4, pp. 66-71, 2003.

[29] Y. X. Chen and Q. Zhao, "On the lifetime of wireless sensor networks," Communications Letters, IEEE, vol. 9, no. 11, pp. 976-978, 2005.

[30] S. Singh, M. Woo, and C. S. Raghavendra, "Power-aware routing in mobile ad hoc networks," in ACM/IEEE Mobile computing and networking. ACM, 1998, pp. 181-190.

[31] R. Guerraoui and E. Ruppert, "Even small birds are unique: Population protocols with identifiers," in Technical Report CSE-2007-04. York University, 2007. 